\title{
Correspondence
}

Contents: Bipolar disorder following a left basal ganglia stroke/Recruitment for post-natal depression studies/Tricyclic-induced seizures and absent ECT response/Alzheimer's disease and Lewy body dementia/Psychiatric morbidity in the relatives of schizophrenic probands/Lithium toxicity at therapeutic serum levels/Catatonia: the tension insanity/ Low serum cholesterol and suicide/Eye-movement desensitisation to overcome post-traumatic stress disorder/How well are 'cured' anorexic nervosa patients?/Psychiatric symptoms in cannabis users/ Fluoxetine - induced mania in a patient with poststroke depression/Relapse following withdrawal of drug addiction/Percutaneous endoscopic gastrostomy and severe endogenous depression.

\section{Bipolar disorder following a left basal-ganglia stroke}

SIR: Typical bipolar disorder (BD) may occur in response to various physical causes such as brain stroke with clinical manifestations remarkably similar to those found in patients with functional BD (Starkstein \& Robinson, Journal, February 1989, 154, 170-182). Many authors have suggested that there is a preponderance of right-sided lesions (Cummings \& Mendez, 1984; Danel et al, 1989) and that the location would be mainly in a limbic or limbic-related area. We report on a patient in whom a BD developed secondary to a left basal-ganglia stroke.

Case report. The patient was a 47-year-old right-handed man who had negative family history of affective disorder or other psychiatric illness. He had no psychiatric symptoms until the age of 46 , when he suddenly developed a manic syndrome characterised by euphoria, grandiose delusions, talkativeness, irritability, insomnia and spending of large amounts of money. He also showed increased libido and activity.

The patient was initially taken to a neurological service where a cranial computerised tomography scan was done and resulted in a left basal-ganglia stroke. He did not present any motor symptoms. The manic episode was treated with a standard neuroleptic and gradually gave rise to a depressive phase characterised by lassitude, depressive mood, apathy, early morning awakening, loss of libido, anorexia and suicidal thoughts. When the patient came to our out-patient service he was at the end of this major depressive episode, presenting mild symptoms. The patient was started on lithium therapy associated to clonazepam and he has remained asymptomatic.

Secondary BD is a relatively rare consequence of stroke because it requires two factors: firstly a predisposing factor of either a genetic loading for affective disorder or subcortical atrophy; and secondly a lesion of a specific right-hemisphere limbic-connected structure (Starkstein \& Robinson, 1989, see above). Despite this, our patient did not have a family history of affective disorder, nor did the lesion affect the right hemisphere. The basal ganglia, as a limbic-related area, may be implicated in cases of this pathology. The interval between acquiring the brain lesion and the onset of mania may be as long as three years (Shukla et al, 1987; Starkstein \& Robinson, 1989, see above); we do not exactly know when the patient had a stroke as he did not have any motor symptoms preceding the manic episode.

Although this case contributes to the concept that the limbic and limbic-related areas are implicated in the aetiology of $\mathrm{BD}$, it also supports the opinion of Jampala \& Abrams (1983) that it is premature to consider mania to be a syndrome of the nondominant hemisphere.

Cummings, J. L. \& Mendez, M. F. (1984) Secondary mania with focal cerebrovascular lesions. American Journal of Psychiatry, 141, 1084-1087.

Danel, T., Comayras, S., Goudemand, M., et al (1989) Troubles de l'humeur et infarctus de l'hèmisphère droit. L'Encéphale, $X V$, 549-553.

JAmpala, V. C. \& ABrams, R. (1983) Mania secondary to left and right hemisphere damage. American Journal of Psychiatry, 140, 1197-1199.

Shukla, S., CoOK, B. L., MUKheruee, S., et al (1987) Mania following head trauma. American Journal of Psychiatry, 144, 93-96.

GUSTAVO TURECKI

Jair De Jesus Mari José Alberto Del Porto

Ambulatório de Distúrbios Afetivos

Departamento de Psiquiatria

Escola Paulista de Medicina

Rua Botucatu, $740-3^{\circ}$ andar

04023-900 - São Paulo

Brazil 